\title{
Clinical and pathological analysis of colonic Crohn's disease, including a subgroup with ulcerative colitis-like features
}

Genevieve Soucy ${ }^{1}$, Helen H Wang ${ }^{2}$, Francis A Farraye ${ }^{3}$, Jason F Schmidt ${ }^{4}$, Alton B Farris ${ }^{5}$, Gregory Y Lauwers ${ }^{5}$, Sandra R Cerda ${ }^{3}$, Kleanthis G Dendrinos ${ }^{6}$ and Robert D Odze ${ }^{7}$

${ }^{1}$ Department of Pathology, Centre Hospitalier Universitaire de Montreal, Montreal, Quebec, Canada;

${ }^{2}$ Department of Pathology, Beth Israel Deaconess Medical Center, Boston, MA, USA; ${ }^{3}$ Department of

Gastroenterology, Boston Medical Center, Boston, MA, USA; ${ }^{4}$ Department of Pathology, Methodist Health

System, Dallas, TX, USA; ${ }^{5}$ Department of Pathology, Massachusetts General Hospital, Boston, MA, USA;

${ }^{6}$ Department of Gastroenterology, Internal Medicine Group, Cheyenne, WY, USA and ${ }^{7}$ Department of

Pathology, Brigham and Women's Hospital, Boston, MA, USA

Little is known regarding the clinical and, in particular, pathological manifestations of patients with isolated colonic Crohn's disease. The purpose of this study was to evaluate the clinical and pathological features of patients with Crohn's disease limited to the colon at initial presentation, and to determine whether there are any histological features that are predictive of outcome after surgery. The clinical features, outcome after surgery, and pathological features of colonic resection specimens of 73 patients who presented initially with isolated colonic Crohn's disease were evaluated and compared with 45 Crohn's disease patients who presented initially with both ileal and colonic involvement. Clinically, patients with isolated colonic Crohn's disease presented at a significantly older age at the time of diagnosis, and had a significantly shorter duration of colitis before surgical resection, than did patients with ileocolonic Crohn's disease at disease onset. Pathologically, patients with isolated colonic Crohn's disease showed a significantly higher proportion of cases with subtotal, total, or leftsided colitis, and significantly fewer strictures/stenosis, pericolonic adhesions, pyloric metaplasia, and cases with proximal worse than distal colonic disease. Overall, patients with isolated colonic Crohn's disease showed a trend toward a lower number of major microscopic Crohn's disease features. A small proportion of patients from both Crohn's disease groups (14\% and $13 \%$, respectively) showed inflammatory disease limited to the mucosa, without mural involvement, reminiscent of ulcerative colitis, and these were termed 'ulcerative colitislike Crohn's disease'. These patients were significantly younger than those with mural involvement. Overall, $44 \%$ of patients from both Crohn's disease groups developed at least one adverse outcome, and neither the number nor the type of major Crohn's disease features correlated with adverse outcome. Patients with isolated colonic involvement have distinctive clinical and pathological features. A small subgroup of Crohn's patients shows only mucosal involvement reminiscent of ulcerative colitis.

Modern Pathology (2012) 25, 295-307; doi:10.1038/modpathol.2011.120; published online 12 August 2011

Keywords: colitis; ileitis; inflammatory bowel disease; pathology; ulcerative colitis

Correspondence: Dr RD Odze, MD, FRCPC, Chief of Gastrointestinal Pathology, Brigham and Women's Hospital, Boston, MA, USA or Professor of Pathology, Harvard Medical School, Brigham and Women's Hospital, Pathology, 75 Francis Street, Boston, MA 02115, USA.

E-mail: rodze@partners.org

This study was presented in part at the United States and Canadian Academy of Pathology annual meeting in Denver, Colorado, 2008.

Received 18 May 2011; revised 13 June 2011; accepted 13 June 2011; published online 12 August 2011
The two most common forms of inflammatory bowel disease are ulcerative colitis and Crohn's disease. In general, ulcerative colitis is considered a mucosal disease, whereas Crohn's disease is both a mucosal and a mural disorder. ${ }^{1,2}$ Ulcerative colitis nearly always involves the rectum before treatment, is diffuse and continuous, and typically shows an absence of Crohn's disease-like histological features, such as granulomas, deep fissuring ulcers, transmural lymphoid aggregates, and small intestine 
involvement. ${ }^{3,4}$ Recent studies have suggested that some cases of ulcerative colitis may show Crohn's disease-like features, such as patchy and segmental disease, early fissuring ulcers (particularly in fulminant cases), granulomas related to ruptured crypts, right-sided appendiceal/cecal involvement in patients with left-sided disease, and small intestine involvement. ${ }^{3,5-10}$ The pathological spectrum, and outcome, of patients with both typical and atypical ulcerative colitis has been well investigated. ${ }^{4}$

In contrast, little is known regarding the pathological spectrum, natural history, and outcome, particularly after surgery, in patients with isolated Crohn's disease of the colon. The incidence of isolated colonic involvement, at disease onset, ranges from $14 \%$ to $32 \%$ of all cases of Crohn's disease. ${ }^{11,12}$ In 2005, a working group of investigators developed an integrated clinical, molecular, and serological classification of inflammatory bowel disease, known as the Montreal classification of inflammatory bowel disease. ${ }^{13}$ In this system, Crohn's disease is classified on the basis of three predominant parameters: age at diagnosis (age below 17 years, 17-40 years, and $>40$ years), anatomical location of disease (ileal, colonic, ileocolonic, and upper GI tract), and behavior (non-stricturing and non-penetrating, stricturing, penetrating). ${ }^{13,14}$ The investigators responsible for the Montreal classification recognized that Crohn's disease isolated to the colon may represent a distinct clinical, pathological, serological, and molecular subtype. Little is known about this type as many previous publications on the subject have included cases with either previous or synchronous ileal involvement. ${ }^{15-17}$ Furthermore, in contrast to ulcerative colitis, Crohn's disease of the colon shows more variable clinical and pathological features. ${ }^{13}$ Although some cases show classic histological features, such as strictures, non-necrotizing granulomas, transmural lymphoid aggregates, segmental and patchy disease, fissures, sinus tracts and fistulas, and perianal disease, others do not. ${ }^{5,18-21}$ In fact, some patients with isolated colonic Crohn's disease show few, if any, classic gross and microscopic features of this disorder, as outlined above, and, thus, resemble ulcerative colitis clinically and histologically. ${ }^{19-21}$ These cases have been termed 'superficial Crohn's disease' or 'ulcerative colitislike Crohn's disease'. Unfortunately, the clinical and pathological features, and natural history, after surgical treatment, of patients with isolated colonic Crohn's disease or ulcerative colitis-like Crohn's disease of the colon are largely unknown. The outcome of patients with isolated colonic Crohn's disease, particularly in patients without cardinal histological features, has been poorly studied, and has never been evaluated systematically in patients in the era of immunomodulator and immunosuppressive therapy. ${ }^{4,11,13}$ Interestingly, a few previous studies have suggested that some patients with disease limited to the colon have a good outcome after colectomy and ileal pouch-anal anastomosis procedure, ${ }^{22-24}$ but at present, histological features that may be predictive of outcome after surgical resection are unknown.

Therefore, the purpose of this study was: (1) to evaluate the clinical and pathological features, and outcome, of a select group of patients with Crohn's disease limited to the colon at initial presentation, who required surgical resection and (2) to determine the characteristics of a small subgroup of these patients, who resemble ulcerative colitis histologically (ulcerative colitis-like Crohn's disease). As a comparison (control) group, patients with both ileal and colonic Crohn's disease at initial presentation, who also had a surgical resection, were included in the study.

\section{Materials and methods}

\section{Study Group}

A total of 118 consecutive patients with involvement of the colon (either with or without ileal involvement) by Crohn's disease at initial presentation were identified (Figures 1 and 2) from the files of the pathology database in the Departments of Pathology at the Brigham and Women's Hospital, Massachusetts General Hospital, and the Boston Medical Center between the years 1987 and 2008. Only patients with involvement of the colon at initial presentation, and who had a surgical resection with follow-up, were included in this study. Patients with isolated ileal Crohn's disease were excluded. All Crohn's disease diagnoses were established by clinicians using previously published clinical and pathological criteria. ${ }^{14}$ As mentioned above, in total, 118 cases were identified, of which 73 presented initially with isolated colonic Crohn's disease. The remaining 45 patients had both ileal and colonic Crohn's disease at initial presentation, and these represented the control group. These patients were selected during the same time period as patients with isolated colonic Crohn's disease, and were collected consecutively. All of the patients, from both the study and the control groups, were evaluated for a wide variety of clinical and pathological features (both gross and microscopic), in a blinded manner without knowledge of the patient's outcome after surgery. Clinical features evaluated included gender and age of the patient at diagnosis, duration of disease from the onset of symptoms to the first colonic operative procedure, and extent of disease assessed both endoscopically and pathologically. The extent of disease was categorized as total, subtotal (portions of right, transverse, and left colon) involvement, left-sided (disease limited to the colon distal to splenic flexure), and right-sided (disease limited to the cecum and/or the ascending colon). The severity of disease was graded as mild, moderate, severe, or 

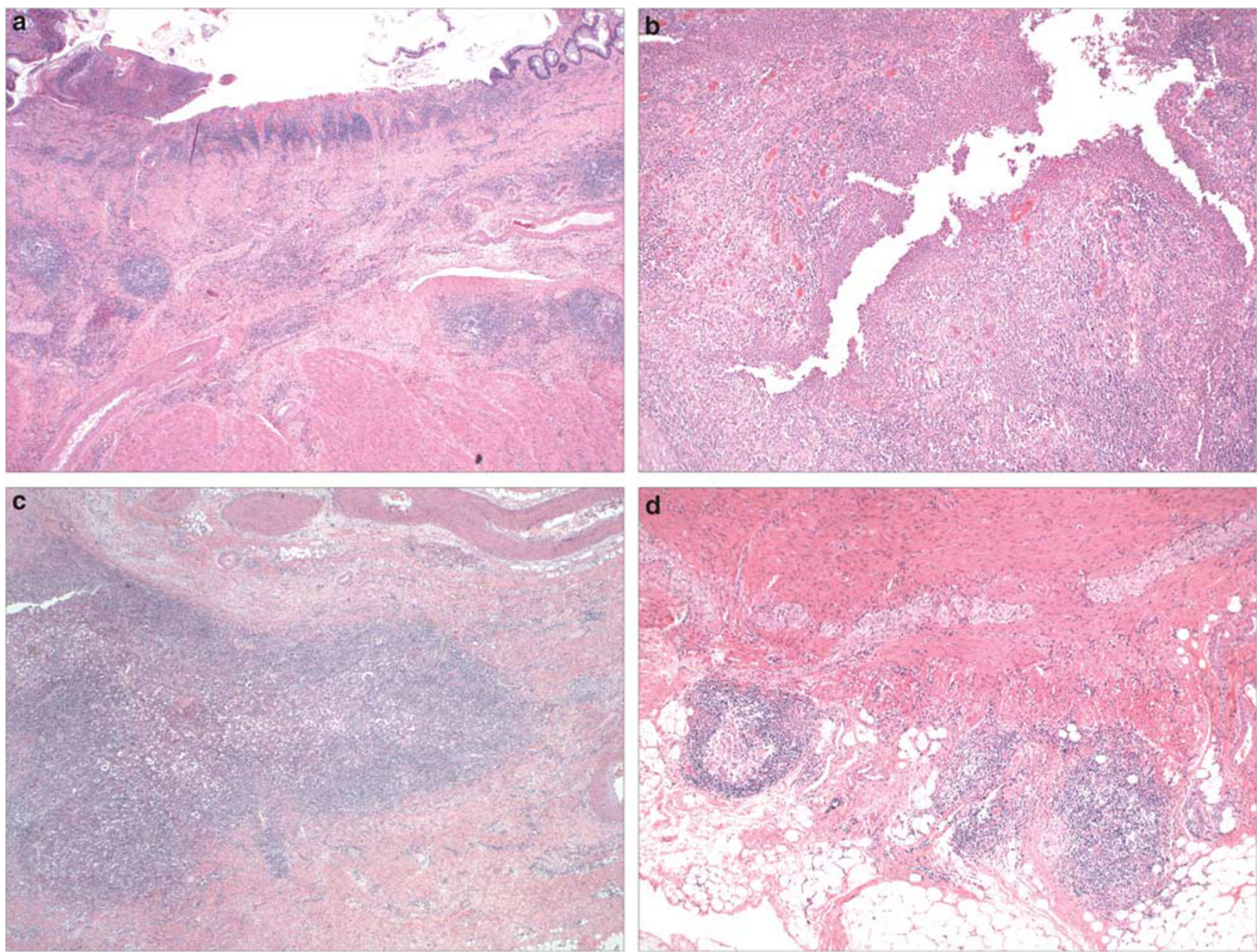

Figure 1 (a-d) Representative images of major histological features in a patient with isolated colonic Crohn's disease. (a) Superficial aspect of bowel wall showing deep ulceration, prominent submucosal inflammation, including lymphoid aggregates, perivascular lymphoid aggregates, and plexitis. (b) Medium power view of a knife-like fissuring ulcer extending from the mucosa into the superficial aspect of the muscularis propria. (c) Medium power view of the deep submucosa showing a sinus tract surrounded by dense lymphoid tissue. (d) Medium power view of the deep aspect of the muscularis propria and subserosa showing lymphoid aggregates and a granuloma (left).

fulminant based on previously published clinical and radiological criteria. ${ }^{13,14}$ The presence or absence of perianal disease (defined by the presence of fissures, fistulas, or abscesses) characteristic of Crohn's disease, either previous to or at the time of the patient's colonic surgery, was also noted.

As mentioned above, all patients had at least one surgical resection of the colon, and the type of procedure (total colectomy, either with or without ileal pouch-anal anastomosis, subtotal colectomy, right hemicolectomy, left hemicolectomy) was recorded. All operations were taken into account when outcome was determined. The medical records of all patients were also reviewed to determine follow-up information and outcome after surgery. More than 40 clinical and outcome features were evaluated in total, but most of these were discarded because they were either inconsistently reported in the medical records, or occurred in too few patients to have meaningful statistics performed. Outcome features considered important to this study included post-operative recurrence (either colonic or non-colonic), type and frequency of pouchitis (categorized as either recurrent antibiotic sensitive or chronic antibiotic resistant), ileal pouch-anal anastomosis pouch (or pouch anastomosis) breakdown that required surgical intervention, development of post-operative fistula, and the need for additional surgical procedures in general. As part of a sub-analysis, patients were also analyzed according to whether they had a 'favorable' or 'unfavorable' outcome based on the absence or presence, respectively, of any one or more of the previously mentioned outcome features. This study was approved by the Institution Review Board at each of the participating institutions.

\section{Pathological Methods}

Patients' colonic resection specimens were evaluated for a wide range of gross and microscopic 

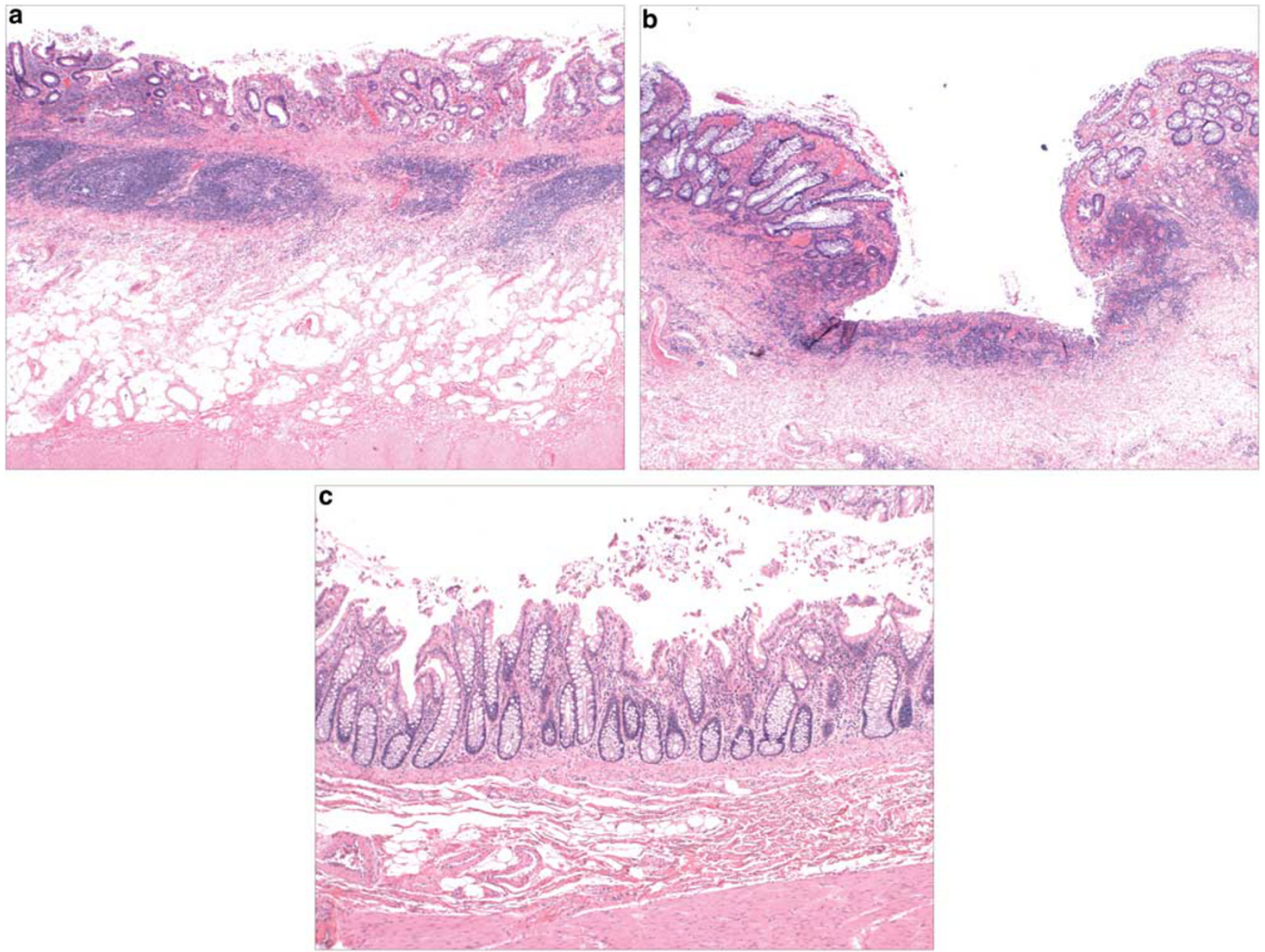

Figure 2 (a-c) Representative images of a patient with ulcerative colitis-like Crohn's disease of the colon. (a) Lower power view of the bowel wall showing moderate chronic active colitis in the mucosa and dense lymphoid tissue in the muscularis mucosa and superficial submucosa, but the remainder of the submucosa and the muscularis propria show no evidence of inflammation in the form of transmural lymphoid aggregates, sinus tracts or fistulas, or serositis. (b) Another area of colonic mucosa from the same patient as panel a showing a mucosal ulcer extending into the superficial submucosa. (c) Medium power view of a section from the rectum in which the mucosa shows evidence of chronic inactive colitis with slight distortion of the crypts, but without active inflammation. It must be noted that there is no evidence of inflammation in the submucosa or muscularis propria.

pathological features. The ileum, in both study and control patients, was also evaluated to confirm the absence or presence of involvement with Crohn's disease in the study and control groups, respectively. The gross features were evaluated by a review of patients' surgical pathology reports, with input from the patient's endoscopy or operative reports, in instances in which particular pieces of information were missing or needed to be clarified. Microscopic evaluation was performed on routinely processed, formalin-fixed, resection specimens by two of the pathologists (GS and RDO) without knowledge of the patient's natural history or outcome.

Gross features evaluated included the presence or absence of stenosis or strictures, adhesions, creeping fat (documented at the time of surgery), sinus or fistula tracts, and segmental disease (noted grossly and confirmed histologically).

Microscopically, $>30$ features were evaluated in each case, and these were separated into 'major' and 'minor' histological features for classification. Major features were designated as such based on their previously reported characteristic association with Crohn's disease of the gastrointestinal tract. These included the following: (1) more severe disease in the proximal, compared with the distal colon, (2) skip lesions (absolute), (3) non-necrotizing granulomas unrelated to ruptured crypts or foreign material, (4) transmural lymphoid aggregates, and (5) deep ulcers penetrating into the muscularis propria (categorized as either fissuring (knife-like penetrating into the muscularis propria) or nonfissuring). All of these criteria were evaluated as per previously published criteria. Skip lesions were defined as absolute when histologically normal (absence of active or chronic disease) mucosa was present in one tissue section, but either active or chronic disease was present both proximal and distal to the location of the normal area of the bowel. For statistical analyses (see below), patients 
were considered to have 'Crohn's disease-like features' if one or more of the following features were present: perianal disease, segmental disease, sinus or fistula tracts, proximal worse than distal disease, absolute skip lesions documented histologically, non-necrotizing granulomas, transmural lymphoid aggregates, or deep fissuring ulceration.

Minor histological features evaluated include the presence or absence of prominent submucosal fibrosis, neural hypertrophy, hypertrophy of the muscularis mucosa and/or muscularis propria, plexitis, serositis, and pyloric metaplasia. All of these features have been associated with Crohn's disease, but are non-specific, and, thus, serve as less 'characteristic' features of Crohn's disease compared with the major features outlined above. Nevertheless, all of these features were evaluated statistically to the same extent as the 'major' features.

\section{Statistical Methods}

The clinical, histological, and outcome parameters for each patient group (isolated colonic Crohn's disease and ileocolonic Crohn's disease) were compared using Stata statistical software (Stata corporation, College Station, TX, USA). Depending on the sample size, either $\chi^{2}$ test or Fisher's extract test was used for categorical data, and either $t$-test or MannWhitney test was used for numerical data. A $P$-value $<0.05$ was considered statistically significant.

\section{Results}

\section{Clinical Features}

Table 1 summarizes the clinical features of patients with isolated colonic Crohn's disease and ileocolonic Crohn's disease at initial presentation. Overall, there were 50 men and 68 women of mean age 33.0 years (range: 8-80 years). A total of 108 were Caucasian and 9 were non-Caucasian. The mean duration of colitis before surgery was 9.7 years (range: 0-44 years). Overall, 12, 14, 32, and 25\% of patients had total, subtotal, left-sided only, or rightsided only colonic disease, respectively, at initial presentation. Overall, $16 \%$ of patients had mild disease, $28 \%$ moderate, $48 \%$ severe, and $7.6 \%$ fulminant at the time of surgery. Overall, the mean follow-up interval after surgery for all patients was 5.9 years (range: 0-21 years). Patients with isolated colonic Crohn's disease and ileocolonic Crohn's disease did not differ with regard to any of the clinical features, except that patients in the former group presented at a significantly older age (37 vs 26 years, $P=0.003$ ) at onset of disease, showed a shorter interval of time from diagnosis to surgery (7.7 vs 13.0 years, $P=0.047$ ), presented more often with subtotal or total colitis (35 vs 11\%) and with left-sided disease (45 vs 11\%), than did patients in the latter group $(P<0.001)$. As a result, a significantly higher percentage of patients with isolated colonic Crohn's disease had a total or subtotal

Table 1 Clinical features of patients with colonic Crohn's disease

\begin{tabular}{|c|c|c|c|c|}
\hline \multirow[t]{2}{*}{ Features } & \multicolumn{3}{|c|}{ Colonic pathology } & \multirow[b]{2}{*}{$\mathrm{P}$-value ${ }^{\mathrm{a}}$} \\
\hline & $\begin{array}{l}\text { Isolated colonic Crohn's } \\
\qquad \mathrm{N}=73\end{array}$ & $\begin{array}{l}\text { Ileocolonic Crohn's } \\
\qquad \mathrm{N}=45\end{array}$ & $\begin{array}{c}\text { Total } \\
\mathrm{N}=118\end{array}$ & \\
\hline $\mathrm{M} / \mathrm{F}$ ratio & $29 / 44(1: 1.5)$ & $21 / 24(1: 1.1)$ & $50 / 68(1: 1.4)$ & 0.46 \\
\hline Mean age (years) & 37 & 26 & 33 & $0.003^{\mathrm{b}}$ \\
\hline Duration of colitis (years) & 7.7 & 13.0 & 9.7 & 0.047 \\
\hline Extent of colitis & & & & $<0.001$ \\
\hline Total & $12 / 73(16 \%)$ & $2 / 45(4.4 \%)$ & $14 / 118(12 \%)$ & \\
\hline Subtotal & 14/73 (19\%) & $3 / 45(6.7 \%)$ & 17/118 (14\%) & \\
\hline Left sided & $33 / 73(45 \%)$ & $5 / 45(11 \%)$ & $38 / 118(32 \%)$ & \\
\hline Right sided & $10 / 73(14 \%)$ & $20 / 45(44 \%)$ & $30 / 118(25 \%)$ & \\
\hline Other & $4 / 73(5.5 \%)$ & $15 / 45(33 \%)$ & $19 / 118(16 \%)$ & \\
\hline Severity & & & & 0.27 \\
\hline Mild & $14 / 73(19 \%)$ & $5 / 45(11 \%)$ & $19 / 118(16 \%)$ & \\
\hline Moderate & $23 / 73(32 \%)$ & $10 / 45(22 \%)$ & $33 / 118(28 \%)$ & \\
\hline Severe & $32 / 73(44 \%)$ & $25 / 45(56 \%)$ & $57 / 118(48 \%)$ & \\
\hline Fulminant & $4 / 73(5.5 \%)$ & $5 / 45(11 \%)$ & 9/118 (7.6\%) & \\
\hline Type of surgery & & & & $<0.001$ \\
\hline Total colectomy & $16 / 73(22 \%)$ & $3 / 45(6.7 \%)$ & 19/118 (16\%) & \\
\hline Subtotal colectomy & $13 / 73(18 \%)$ & $2 / 45(4.4 \%)$ & $15 / 118(13 \%)$ & \\
\hline Left hemicolectomy & $31 / 73(42 \%)$ & $11 / 45(24 \%)$ & $42 / 118(36 \%)$ & \\
\hline Right hemicolectomy & $11 / 73(15 \%)$ & $25 / 45(56 \%)$ & $36 / 118(31 \%)$ & \\
\hline Ileal pouch-anal anastomosis & $20 / 73(27 \%)$ & $10 / 45(22 \%)$ & $30 / 118(25 \%)$ & 0.53 \\
\hline Mean follow-up interval (years) & 5.1 & 6.9 & 5.9 & $0.10^{\mathrm{b}}$ \\
\hline
\end{tabular}

${ }^{\mathrm{a}} P$-values were determined by $\chi^{2}$ unless otherwise specified.

${ }^{\mathrm{b}} P$-values were determined by $t$-test. 
colectomy $(40 \%)$ or left hemicolectomy $(42 \%)$ vs patients with ileocolonic Crohn's disease (11 and $24 \%$, respectively, $P<0.001$ ).

\section{Pathological Features}

A summary of the major (gross and microscopic) and minor features of the patient groups are outlined in Tables 2a and b, respectively. In total, strictures or luminal stenosis, adhesions, creeping fat, fistula tract, sinus tract, gross evidence of segmental disease (confirmed histologically), and perianal disease either before or at the time of colon resection were noted in $38,30,29,17,27,52$, and $20 \%$, respectively, of all patients with colonic Crohn's disease (both isolated colonic Crohn's disease and ileocolonic Crohn's disease groups combined). With regard to these gross features, patients with isolated colonic Crohn's disease at initial presentation showed significantly fewer cases with strictures or stenosis $(P=0.001)$, adhesions $(P<0.001)$, and segmental disease $(P=0.01)$, than did patients with ileocolonic Crohn's disease. However, none of the other gross features were significantly different between the two patient groups.

Microscopically, major pathological features, such as proximal worse than distal disease, absolute skip lesions, granulomas, transmural lymphoid aggregates, and fissuring ulcers were present in $28,16,52$,
71 , and $42 \%$, respectively, of all colonic Crohn's disease patients. Overall, the proportion of patients with each of these histological features was similar between the two patient groups, except for the finding of proximal worse than distal disease, which was significantly less common in patients with isolated colonic Crohn's disease vs ileocolonic Crohn's disease at initial presentation (18 vs 44\%, $P=0.004$ ).

A list of the minor pathological features and their frequencies in each of the patient groups is indicated in Table 2b. None of the minor pathological features showed significant differences in frequency between patients with isolated colonic Crohn's disease compared with those with ileocolonic Crohn's disease, except for perivascular lymphoid aggregates, which were significantly more common in patients with isolated colonic Crohn's disease $(42 \%)$, and pyloric metaplasia, which was significantly less common in this patient group $(4.0 \%)$, compared with patients with ileocolonic Crohn's disease (perivascular aggregates, 22\%; pyloric metaplasia, 73\%).

A separate statistical analysis was performed in which the number and type of major and minor features were evaluated and compared between the study and control groups. In this analysis, a trend $(P=0.08)$ toward a lower overall number of major gross and major microscopic features in patients

Table 2 (a) Major pathological and (b) Minor pathologic features of patients with colonic Crohn's disease

\begin{tabular}{|c|c|c|c|c|}
\hline \multirow[t]{2}{*}{ Features } & \multicolumn{3}{|c|}{ Colonic pathology } & \multirow[b]{2}{*}{ P-value ${ }^{\mathrm{a}}$} \\
\hline & $\begin{array}{l}\text { Isolated colonic Crohn's } \\
\qquad \mathrm{N}=73\end{array}$ & $\begin{array}{l}\text { Ileocolonic Crohn's } \\
\qquad \mathrm{N}=45\end{array}$ & $\begin{array}{c}\text { Total } \\
\mathrm{N}=118\end{array}$ & \\
\hline \multicolumn{5}{|l|}{ (a) Major } \\
\hline \multicolumn{5}{|l|}{ Gross } \\
\hline Stricture/stenosis & $19 / 72(26 \%)$ & $26 / 45(58 \%)$ & $45 / 117(38 \%)$ & 0.001 \\
\hline Adhesions & $10 / 72(14 \%)$ & $25 / 45(56 \%)$ & $35 / 117(30 \%)$ & $<0.001$ \\
\hline Creeping fat & $19 / 72(26 \%)$ & $15 / 45(33 \%)$ & $34 / 117(29 \%)$ & 0.42 \\
\hline Fistula tract & $9 / 73(12 \%)$ & $11 / 45(24 \%)$ & $20 / 118(17 \%)$ & 0.09 \\
\hline Sinus tract & $21 / 73(29 \%)$ & $11 / 45(24 \%)$ & $32 / 118(27 \%)$ & 0.61 \\
\hline Segmental disease & $30 / 70(43 \%)$ & $30 / 45(67 \%)$ & $60 / 115(52 \%)$ & 0.01 \\
\hline Perianal disease & $18 / 73(25 \%)$ & $6 / 45(13 \%)$ & $24 / 118(20 \%)$ & 0.14 \\
\hline \multicolumn{5}{|l|}{ Microscopic } \\
\hline Proximal > distal (severity) & $13 / 73(18 \%)$ & $20 / 45(44 \%)$ & $33 / 118(28 \%)$ & 0.004 \\
\hline Skip lesions (absolute) & $10 / 73(14 \%)$ & $9 / 45(20 \%)$ & $19 / 118(16 \%)$ & 0.37 \\
\hline Granulomas & $39 / 73(53 \%)$ & $22 / 45(49 \%)$ & $61 / 118(52 \%)$ & 0.63 \\
\hline Transmural lymphoid aggregates & $49 / 73(67 \%)$ & $35 / 45(78 \%)$ & $84 / 118(71 \%)$ & 0.22 \\
\hline Fissuring ulcers & $29 / 73(40 \%)$ & $20 / 45(44 \%)$ & $49 / 118(42 \%)$ & 0.61 \\
\hline \multicolumn{5}{|l|}{ (b) Minor } \\
\hline \multicolumn{5}{|l|}{ Microscopic } \\
\hline Submucosal fibrosis & $64 / 73(88 \%)$ & $36 / 45(80 \%)$ & $100 / 118(85 \%)$ & 0.26 \\
\hline Neural hypertrophy & $31 / 73(42 \%)$ & $17 / 45(38 \%)$ & $48 / 118(41 \%)$ & 0.62 \\
\hline Muscularis mucosae hypertrophy & $28 / 73(38 \%)$ & $22 / 45(49 \%)$ & $50 / 118(42 \%)$ & 0.26 \\
\hline Muscularis propria hypertrophy & $35 / 73(48 \%)$ & $15 / 45(33 \%)$ & $50 / 118(42 \%)$ & 0.12 \\
\hline Plexitis & $23 / 73(32 \%)$ & $9 / 45(20 \%)$ & $32 / 118(27 \%)$ & 0.17 \\
\hline Perivascular lymphoid aggregates & $30 / 72(42 \%)$ & $10 / 45(22 \%)$ & $40 / 117(34 \%)$ & 0.03 \\
\hline Serositis & $35 / 73(48 \%)$ & $29 / 45(64 \%)$ & $64 / 118(54 \%)$ & 0.08 \\
\hline Pyloric metaplasia & $2 / 50(4.0 \%)$ & $30 / 41(73 \%)$ & $32 / 91(35 \%)$ & $<0.001$ \\
\hline
\end{tabular}

\footnotetext{
${ }^{\mathrm{a}} P$-values were determined by $\chi^{2}$ test.
} 
with isolated colonic Crohn's disease compared with those with ileocolonic Crohn's disease was observed. For instance, $56 \%$ of patients with isolated colonic Crohn's disease had $\leq 3$ major Crohn's disease features compared with only $26 \%$ of the colons in patients with ileocolonic Crohn's disease. An evaluation of only the major gross features revealed a statistically significant lower number of gross Crohn's disease features in isolated colonic Crohn's disease $(74 \% \leq 2$ Crohn's disease features) compared with ileocolonic Crohn's disease (45\%, $P=0.03$ ). However, no significant differences were observed between the two patient groups when only the number of major or minor microscopic features were compared between the two patient groups ( $P>0.05$, respectively).

\section{Outcome}

Table 3 summarizes the outcome data for patients in this study. The mean follow-up interval, after surgery, was 5.9 years (isolated colonic Crohn's disease, 5.1 years; ileocolonic Crohn's disease, 6.9 years). Overall, $39 \%$ of patients developed recurrent disease post-operatively $(28 \%$ colonic recurrence only, $10 \%$ non-colonic recurrence only, and $6 \%$ with both colonic and non-colonic recurrence). Overall, 20 patients with isolated colonic Crohn's disease and 10 patients with ileocolonic Crohn's disease had an ileal pouch-anal anastomosis ('pouch') procedure because of either a mistaken diagnosis of ulcerative colitis preoperatively or a diagnosis of indeterminate colitis. In all of these instances, a final diagnosis of Crohn's disease was made by analysis of the resection specimen. Overall, $27 \%$ showed recurrent antibiotic-responsive pouchitis. Chronic pouchitis developed in a smaller proportion of patients (17\%), and no patient had pouch anastomosis breakdown. Finally, $18 \%$ of patients had post-operative fistula formation. Thus, overall, $44 \%$ of patients had at least one of the above-mentioned adverse outcomes, whereas 56\% had no adverse outcome. Although not necessarily regarded as an adverse outcome, overall, $34 \%$ demonstrated a need for at least one additional surgical procedure, but in many of these instances, the surgical procedures were due to non-pathological sequelae, such as revision of scars and stoma reversal. A comparison of patients with isolated colonic Crohn's disease vs those with ileocolonic Crohn's disease at initial presentation revealed no significant differences in any of the specific adverse outcomes, or regarding the percentage of patients with at least one adverse outcome, between the two patient groups.

A separate statistical analysis was performed in which the number and type of major and minor features were correlated with outcome in each of the study and control groups separately or combined. Overall, there was no correlation between either the number or any specific type of major Crohn's disease features, and the development of at least one adverse outcome for both patient groups combined, or for the individual patient groups when those were analyzed separately, with one exception. Younger patient age was associated with a significantly higher risk of development of an adverse outcome in patients with ileocolonic Crohn's disease, but not in patients with isolated colonic Crohn's disease. Patients with ileocolonic Crohn's disease who developed at least one adverse outcome had a mean age of 20 vs 32 years for those who did not develop any adverse outcome $(P=0.01)$.

\section{Ulcerative-Colitis-Like Crohn's Disease}

Overall, 10 of 73 patients with isolated colonic Crohn's disease $(14 \%)$, and the colon in 6 of 45 patients with ileocolonic Crohn's disease (13\%), were categorized as ulcerative colitis-like Crohn's disease' based on the presence of disease limited to

Table 3 Outcome of patients with colonic Crohn's disease

\begin{tabular}{|c|c|c|c|c|}
\hline Outcome & $\begin{array}{l}\text { Isolated colonic Crohn's } \\
\qquad \mathrm{N}=73\end{array}$ & $\begin{array}{l}\text { Ileocolonic Crohn's } \\
\qquad \mathrm{N}=45\end{array}$ & $\begin{array}{c}\text { Total } \\
\mathrm{N}=118\end{array}$ & P-value ${ }^{a}$ \\
\hline \multicolumn{5}{|l|}{ Recurrence } \\
\hline Colonic & $18 / 63(29 \%)$ & $10 / 34(29 \%)$ & $28 / 97(29 \%)$ & 0.93 \\
\hline Non-colonic & $9 / 63(14 \%)$ & $7 / 34(21 \%)$ & $16 / 97(16 \%)$ & 0.43 \\
\hline \multicolumn{5}{|l|}{ Pouchitis } \\
\hline Recurrent & $6 / 19(30 \%)$ & $2 / 10(20 \%)$ & $8 / 30(27 \%)$ & $0.68^{\mathrm{b}}$ \\
\hline Chronic/resistant & $3 / 20(15 \%)$ & $2 / 10(20 \%)$ & $5 / 30(17 \%)$ & $1.0^{\mathrm{b}}$ \\
\hline Pouch anastomotic breakdown & $0 / 20(0 \%)$ & $0 / 10(0 \%)$ & $0 / 30(0 \%)$ & \\
\hline Fistula & $11 / 73(15 \%)$ & $10 / 45(22 \%)$ & $21 / 118(18 \%)$ & 0.32 \\
\hline Total with adverse outcome & $32 / 73(44 \%)$ & $20 / 45(44 \%)$ & $52 / 118(44 \%)$ & 0.95 \\
\hline Need for additional procedures & $20 / 61(33 \%)$ & $10 / 26(38 \%)$ & $30 / 87(34 \%)$ & 0.61 \\
\hline
\end{tabular}

${ }^{\mathrm{a}} P$-values were determined by $\chi^{2}$ test unless otherwise specified.

${ }^{\mathrm{b}} P$-values were determined by Fisher's exact test. 
the colonic mucosa, without mural involvement in the form of deep fissuring ulcers, transmural lymphoid aggregates, sinus tracts, or fistulas (Table 4). Of these 16 patients, 7 were men and 9 were women, of mean age 23 years (range: 11-51 years). The mean duration of colitis before surgery was 11 years (range: 0-30 years). Eight patients $(50 \%)$ showed isolated left-sided colonic involvement, whereas one showed subtotal and two total colon involvement. In three patients, the right colon was the only segment of colon involved. Most patients showed mild-to-moderate disease (10/16, $63 \%$ ); none had fulminant disease. No significant differences were noted with regard to the clinical features between isolated Crohn's disease and ileocolonic Crohn's disease patients with ulcerative colitis-like Crohn's disease in the colon.

Pathologically (Table 5), three of the patients (20\%) had adhesions, three had creeping fat $(20 \%)$, and six $(40 \%)$ had segmental disease, but none showed strictures or stenosis. By definition, none had fistulas or sinus tracts, transmural lymphoid aggregates, or fissuring ulcers detected at the time of surgery, but two (13\%) had perianal disease. Microscopically, four patients (25\%) showed more severe disease in the proximal, compared with the distal colon, three $(19 \%)$ showed absolute skip lesions, and seven (44\%) showed granulomas. Table 5 also lists the frequency of minor histological features in ulcerative colitis-like Crohn's disease patients.

In a separate comparison, none of the clinical or (non-mural) pathological features were significantly more, or less, frequent in patients with ulcerative colitis-like colonic Crohn's disease compared with patients with non-ulcerative colitis-like colonic Crohn's disease. However, the mean age at diagnosis was significantly younger in all ulcerative colitislike Crohn's disease patients (23 years) compared with all non-ulcerative colitis-like Crohn's disease patients (35 years; $P=0.02$ ).

Upon follow-up (mean follow-up interval: 5.7 years), overall, 9 of the 16 patients (56\%) showed an adverse outcome (Table 6). Four had only colonic, one had only non-colonic (ileal), and two had both colonic and non-colonic recurrence. Two patients in each group had an ileal pouch-anal anastomosis procedure, and two of these had recurrent antibiotic-responsive pouchitis. One patient $(6 \%)$ revealed chronic pouchitis and four had fistula formation, but none showed pouch, or pouch anastomosis, breakdown. No association was noted between any particular gross or microscopic feature, or between the number of Crohn's disease features, and adverse outcome, in ulcerative colitislike colonic Crohn's disease in patients with either isolated colonic or ileocolonic Crohn's disease, or when both groups were combined in the analysis.

Table 4 Clinical features of patients with ulcerative colitis-like ${ }^{a}$ Crohn's disease of the colon

\begin{tabular}{|c|c|c|c|}
\hline \multirow[t]{2}{*}{ Features } & \multicolumn{3}{|c|}{ Ulcerative colitis-like Crohn's diesase } \\
\hline & $\begin{array}{l}\text { Isolated colonic }{ }^{\mathrm{b}} \text { Crohn's } \\
\mathrm{N}=10\end{array}$ & $\begin{array}{l}\text { Ileocolonic } \text { Crohn's }^{\mathrm{b}} \\
\mathrm{N}=6\end{array}$ & $\begin{array}{l}\text { Total } \\
\mathrm{N}=16\end{array}$ \\
\hline $\mathrm{M} / \mathrm{F}$ ratio & $4 / 6(1: 1.5)$ & $3 / 3(1: 1)$ & $7 / 9(1: 1.3)$ \\
\hline Mean age (years) & 24 & 22 & 23 \\
\hline Duration of colitis & 4.6 & 10 & 11 \\
\hline \multicolumn{4}{|l|}{ Extent of colitis } \\
\hline Total & $1 / 10(10 \%)$ & $1 / 6(17 \%)$ & $2 / 16(13 \%)$ \\
\hline Subtotal & $1 / 10(10 \%)$ & 0 & $1 / 16(6.3 \%)$ \\
\hline Left sided & $6 / 10(60 \%)$ & $2 / 6(33 \%)$ & $8 / 16(50 \%)$ \\
\hline Right sided & $2 / 10(20 \%)$ & $1 / 6(17 \%)$ & $3 / 16(19 \%)$ \\
\hline Other & 0 & $2 / 6(33 \%)$ & $2 / 16(13 \%)$ \\
\hline \multicolumn{4}{|l|}{ Severity } \\
\hline Mild & $2 / 10(20 \%)$ & $2 / 6(33 \%)$ & $4 / 16(25 \%)$ \\
\hline Moderate & $4 / 10(40 \%)$ & $2 / 6(33 \%)$ & 6/16 (38\%) \\
\hline Severe & $4 / 10(40 \%)$ & $2 / 6(33 \%)$ & $6 / 16(38 \%)$ \\
\hline Fulminant & 0 & 0 & $0 / 16$ \\
\hline \multicolumn{4}{|l|}{ Type of surgery } \\
\hline Total colectomy & $1 / 10(10 \%)$ & $1 / 6(17 \%)$ & $2 / 16(13 \%)$ \\
\hline Subtotal colectomy & $1 / 10(10 \%)$ & 0 & $1 / 16(6.3 \%)$ \\
\hline Left hemicolectomy & $6 / 10(60 \%)$ & $3 / 6(50 \%)$ & $9 / 16(56 \%)$ \\
\hline Right hemicolectomy & $2 / 10(20 \%)$ & $1 / 6(17 \%)$ & $3 / 16(19 \%)$ \\
\hline Ileal pouch-anal anastomosis & $2 / 10(20 \%)$ & $2 / 6(33 \%)$ & $4 / 16(25 \%)$ \\
\hline Mean follow-up interval (years) & 4.5 & 7.5 & 5.7 \\
\hline
\end{tabular}

${ }^{\mathrm{a}}$ Patients without any of the following: stricture, fissuring ulcer, transmural lymphoid aggregates, fistula, or sinus tract are shown here.

${ }^{\mathrm{b}} \mathrm{P}>0.05$ for all comparisons. 
Table 5 Major and minor pathological features of patients with ulcerative colitis-like ${ }^{\text {a }}$ Crohn's disease of colon

\begin{tabular}{|c|c|c|c|}
\hline \multirow[t]{2}{*}{ Features } & \multicolumn{3}{|c|}{ Ulcerative colitis-like Crohn's disease } \\
\hline & $\begin{array}{l}\text { Isolated colonic }{ }^{\mathrm{b}} \text { Crohn's } \\
\qquad \mathrm{N}=10\end{array}$ & $\begin{array}{l}\text { Ileocolonic }^{\mathrm{b}} \text { Crohn's } \\
\mathrm{N}=6\end{array}$ & $\begin{array}{c}\text { Total } \\
\mathrm{N}=16\end{array}$ \\
\hline \multicolumn{4}{|l|}{ Major } \\
\hline \multicolumn{4}{|l|}{ Gross } \\
\hline Stricture/stenosis & $\mathrm{b}$ & $\mathrm{b}$ & b \\
\hline Adhesions $^{\mathrm{c}}$ & $0 / 9$ & $3 / 6(50 \%)$ & $3 / 15(20 \%)$ \\
\hline Creeping fat & $2 / 9(22 \%)$ & $1 / 6(17 \%)$ & $3 / 15(20 \%)$ \\
\hline Fistula tract & $\mathrm{b}$ & b & b \\
\hline Sinus tract & b & $\mathrm{b}$ & $\mathrm{b}$ \\
\hline Segmental disease & $4 / 9(44 \%)$ & $2 / 6(33 \%)$ & $6 / 15(40 \%)$ \\
\hline Perianal disease & $2 / 10(20 \%)$ & 0 & $2 / 16(13 \%)$ \\
\hline \multicolumn{4}{|l|}{ Microscopic } \\
\hline Proximal > distal (severity) & $1 / 10(10 \%)$ & $3 / 6(50 \%)$ & $4 / 16(25 \%)$ \\
\hline Skip lesions (absolute) & $1 / 10(10 \%)$ & $2 / 6(33 \%)$ & $3 / 16(19 \%)$ \\
\hline Granulomas & $4 / 10(40 \%)$ & $3 / 6(50 \%)$ & $7 / 16(44 \%)$ \\
\hline Transmural lymphoid aggregates & $\mathrm{b}$ & b & b \\
\hline Fissuring ulcers & $\mathrm{b}$ & $\mathrm{b}$ & $\mathrm{b}$ \\
\hline \multicolumn{4}{|l|}{ Minor } \\
\hline Submucosal fibrosis & $0 / 10(0 \%)$ & $4 / 6(67 \%)$ & $13 / 16(81 \%)$ \\
\hline Neural hypertrophy & $2 / 10(20 \%)$ & $2 / 6(33 \%)$ & $4 / 16(25 \%)$ \\
\hline Muscularis mucosae hypertrophy & $5 / 10(50 \%)$ & $2 / 6(33 \%)$ & $7 / 16(44 \%)$ \\
\hline Muscularis propria hypertrophy & $0 / 10(0 \%)$ & $2 / 6(33 \%)$ & $5 / 16(31 \%)$ \\
\hline Plexitis & $1 / 10(10 \%)$ & $0 / 6(0 \%)$ & $1 / 16(6.3 \%)$ \\
\hline Perivascular lymphoid aggregates & $4 / 10(40 \%)$ & $1 / 6(17 \%)$ & $5 / 16(31 \%)$ \\
\hline Serositis & $4 / 10(40 \%)$ & $2 / 6(33 \%)$ & $6 / 16(38 \%)$ \\
\hline Pyloric metaplasia & $1 / 6(17 \%)$ & $3 / 4(75 \%)$ & $4 / 10(40 \%)$ \\
\hline
\end{tabular}

${ }^{\mathrm{a}}$ Patients without any of the following: stricture, fissuring ulcer, transmural lymphoid aggregates, fistula, or sinus tract are shown here.

${ }^{\mathrm{b}} \mathrm{By}$ definition, ulcerative colitis-like colonic Crohn's disease patients do not have these features.

${ }^{\mathrm{C}}$ Isolated colonic vs ileocolonic Crohn's disease; $P=0.04$ by Fisher's exact test; $P>0.05$ for all other comparisons.

Table 6 Outcome of patients with ulcerative colitis-like Crohn's disease of colon ${ }^{\mathrm{a}}$

\begin{tabular}{|c|c|c|c|}
\hline \multirow[t]{2}{*}{ Features } & \multicolumn{3}{|c|}{ Ulcerative colitis-like Crohn's disease } \\
\hline & $\begin{array}{l}\text { Isolated colonic } \text { Crohn's }^{\mathrm{b}} \\
\mathrm{N}=10\end{array}$ & $\begin{array}{l}\text { Ileocolonic }^{\mathrm{b}} \text { Crohn's } \\
\mathrm{N}=6\end{array}$ & $\begin{array}{c}\text { Total } \\
\mathrm{N}=16\end{array}$ \\
\hline \multicolumn{4}{|l|}{ Recurrence } \\
\hline Colonic & $3 / 10(30 \%)$ & $3 / 5(60 \%)$ & $6 / 15(40 \%)$ \\
\hline Non-colonic & $2 / 10(20 \%)$ & $1 / 5(20 \%)$ & $3 / 15(20 \%)$ \\
\hline \multicolumn{4}{|l|}{ Pouchitis } \\
\hline Recurrent & $2 / 2(100 \%)$ & 0 & $2 / 4(50 \%)$ \\
\hline Chronic/resistant & $1 / 1(100 \%)$ & 0 & $1 / 3(33 \%)$ \\
\hline Pouch anastomotic breakdown & $0 / 2$ & $0 / 2$ & $0 / 4$ \\
\hline Fistula & $1 / 10(10 \%)$ & $3 / 6(50 \%)$ & $4 / 16(25 \%)$ \\
\hline Total with adverse outcome & $5 / 10(50 \%)$ & $4 / 6(66 \%)$ & $9 / 16(56 \%)$ \\
\hline Need for additional procedures & $1 / 7(14 \%)$ & $3 / 4(75 \%)$ & $4 / 11(36 \%)$ \\
\hline
\end{tabular}

${ }^{a}$ Patients without any of the following: stricture, fissuring ulcer, transmural lymphoid aggregates, fistula, or sinus tract are shown here.

${ }^{\mathrm{b}} P>0.05$ for all comparisons.

In addition, no significant differences were observed in the frequency, or type, of outcome in ulcerative colitis-like colonic Crohn's disease patients $(N=16)$ compared with non-ulcerative colitis-like colonic Crohn's disease patients $(N=112)$.

\section{Discussion}

Recent clinical and molecular data support the existence of discrete subtypes of Crohn's disease, which are, in part, defined by the predominant 
anatomical location of disease. ${ }^{13,14}$ These subtypes were defined most recently by an inflammatory bowel disease working party in Montreal, Canada, in 2005, as part of the World Congress of Gastroenterology. ${ }^{13}$ The subtypes defined by this working party are isolated terminal ileal disease (L1), isolated colonic disease (L2), combined ileal and colonic disease (L3), and upper GI disease (L4). ${ }^{13,14}$ Unfortunately, little is known regarding the clinical, epidemiological, and in particular, pathological manifestations of patients with isolated colonic Crohn's disease, and this is in part related to the fact that most previous studies on colonic Crohn's disease have not accurately distinguished patients with isolated colonic from those with combined ileal and colonic inflammation. ${ }^{15-17}$ Furthermore, the natural history of isolated colonic Crohn's disease patients, after resection, is largely unknown.

The purpose of this study was to evaluate the clinical and pathological features, and outcome, of patients with Crohn's disease limited to the colon at initial presentation in comparison with patients with ileocolonic Crohn's disease, and to determine the clinical and pathological characteristics of a subgroup of colonic Crohn's disease patients in whom the pathological manifestations of the colon resemble ulcerative colitis histologically because of the absence of mural involvement. Clinically, our results showed that patients with isolated colonic Crohn's disease presented at a significantly older age at diagnosis (37 years) and had a significantly shorter duration of colitis before surgical resection, compared with patients with ileocolonic Crohn's disease at disease onset (mean duration of colitis, 7.7 vs 13 years; $P=0.05$ ). Pathologically, several distinct differences were noted between patients with isolated colonic vs ileocolonic Crohn's disease. Patients with isolated colonic Crohn's disease showed a significantly higher proportion of cases with subtotal or total colitis, or left-side colitis, compared with patients with ileocolonic Crohn's disease who showed predominantly right-sided colonic disease. In addition, patients with isolated colonic Crohn's disease showed significantly fewer strictures/stenosis, and pericolonic adhesions, and a significantly lower number of cases with more severe disease in the proximal than in the distal colon. Perivascular lymphoid aggregates and pyloric metaplasia were also significantly more and less common, respectively, in patients with isolated colonic Crohn's disease compared with ileocolonic Crohn's disease. Although not statistically significant, patients with isolated colonic Crohn's disease showed a trend toward an overall lower number of major gross and microscopic Crohn's disease features. Finally, a small proportion of patients from both groups (14 and $13 \%$, respectively), showed inflammatory changes limited to the mucosa typical of ulcerative colitis (UC-like Crohn's disease). These patients were significantly younger than those with classic Crohn's disease with mural involvement.
Overall, no differences were noted with regard to the development of adverse outcomes between patients with isolated colonic vs those with ileocolonic Crohn's disease at initial presentation, or between patients with ulcerative colitis-like Crohn's disease $v s$ those with mural involvement. On the basis of these results, we conclude that patients with isolated colonic Crohn's disease at initial presentation reveal discrete clinical and pathological features, and this supports the Montreal classification of Crohn's disease.

Our study is the first to perform a detailed pathological analysis of isolated colonic Crohn's disease, and to compare the features directly with ileocolonic Crohn's disease at initial presentation. However, several other predominantly clinicalbased studies have evaluated patients with isolated colonic Crohn's disease, and in some of those studies, less comprehensive pathological analyses were performed. ${ }^{25-27}$ For instance, in 2003, Morpurgo et $a l^{25}$ evaluated the clinical and (selected) pathology features, and outcome after surgery, of 92 patients with Crohn's disease of the colon at initial presentation. In that study, patients were specifically separated into two broad categories based on the presence or absence of granulomas. Patients with granulomas or segmental disease at presentation showed a significantly higher recurrence rate after surgery than did patients without granulomas or those with pancolitis. In addition, similar to the results of our current study, these authors noted that the majority of patients with pancolitis (81\%) had isolated colonic involvement at initial presentation compared with patients with segmental colitis $(37 \%)$ who, in contrast, revealed a higher prevalence rate of extracolonic involvement. Unfortunately, the results of the study by Morpurgo et al are difficult to compare with those of ours because of differences in study design and entry criteria. For instance, they included some patients with previous surgery and those with extracolonic disease at initial presentation. Furthermore, a detailed pathological evaluation of Crohn's disease-like features in the colon and a comparison with the colon in patients with ileocolonic Crohn's disease was not performed.

In the only other study that evaluated patients with isolated colonic Crohn's disease specifically, Hancock et $a l^{26}$ investigated the association of certain genetic polymorphisms with disease location and behavior. In that study of 135 patients, isolated colonic Crohn's disease was more common in females and non-smokers, and showed a statistically higher prevalence rate of the DRB $1{ }^{*} 0103$ allele compared with Crohn's disease patients without colonic involvement. Unfortunately, in that study, only $20.7 \%$ of patients had a colonic resection, and in none was a pathological analysis performed. However, the authors did note that patients with isolated colonic Crohn's disease showed fewer strictures than did patients without colonic involvement (3.7 vs 26\%). Our study documented a lower 
prevalence rate of strictures in the colon of patients with isolated Crohn's disease compared with the colon of patients with ileocolonic Crohn's disease (26 vs 58\%), despite the fact that our overall stricture rate $(38 \%)$ was higher than that observed in the study by Hancock et al. One potential reason for this discrepancy may be due to the fact that all patients in our cohort had undergone a colonic resection, which may have resulted in bias toward patients with more severe disease, as stricture formation is a major indication for surgery in patients with Crohn's disease. Interestingly, Hancock et $a l^{26}$ reported that colonic Crohn's disease patients with the DRB1*0103 allelic polymorphism were associated with a shorter interval of time between the patient's diagnosis and surgery, a result that we also noted in our patients with isolated colonic Crohn's disease patients in comparison with those with ileocolonic Crohn's disease.

Nevertheless, the results of both of these studies, including those of our current study, support the emerging theory that there are discrete biological subtypes of Crohn's disease defined by disease location. ${ }^{14}$ There is other evidence to support this theory. ${ }^{13}$ For instance, aside from differences in clinical and demographic characteristics, and risk factors such as smoking (which shows a higher prevalence rate in patients with ileal disease), others have noted various other differences in pathology, such as a lower (or higher) incidence of perianal disease, and a lower incidence of stenosis and penetrating/fistulating disease, in patients with isolated colonic Crohn's disease. ${ }^{13,26,28-32}$ Aside from the HLA DRB1*0103 results noted by Hancock et $a l,{ }^{26}$ other Crohn's disease susceptibility loci, such as NOD 2, inflammatory bowel disease 5, NOD 1, Isle 23R, and ATC16L1 have also been shown to be associated with Crohn's disease. ${ }^{13,33-37}$ Unfortunately, none of these molecular studies correlated the presence of polymorphisms, or mutations, with specific pathological subtypes of Crohn's disease.

Evidence in favor of colonic Crohn's disease representing a specific clinicopathological subtype stems from results of outcome studies as well. For instance, in the study noted above by Hancock et $a l,{ }^{26}$ the cumulative risk of surgery for patients with colonic Crohn's disease was $32.8 \%$ at 10 years vs $90 \%$ for patients with ileal disease. In fact, in some studies, the risk of surgery for patients with colonic disease approaches the rates reported for patients with ulcerative colitis. ${ }^{22-24}$ Other studies have noted a higher risk of symptomatic recurrence of patients with Crohn's disease of the colon. ${ }^{38,39}$ For instance, in a population-based cohort study of 833 patients with Crohn's colitis by Bernell et al, the cumulative 10-year risk of symptomatic recurrence was 58 and $47 \%$, respectively, after colectomy with ileorectal anastomosis and segmental colonic resection, respectively. ${ }^{38}$ In that study, the presence of perianal disease, ileorectal anastomosis, and segmental resection were independent risk factors for postoperative recurrence. Other studies have shown that some pathological features, such as granulomas, may be associated with recurrence after surgery; however, this is controversial. ${ }^{40-42}$ Furthermore, other than the study by Morpurgo et $a{ }^{25}$ these studies did not evaluate isolated colonic Crohn's disease specifically.

In our current study, we did not note any significant difference in the development of adverse outcomes in patients with isolated colonic Crohn's disease compared with patients with ileocolonic Crohn's disease at initial presentation. Overall, 44\% of patients with isolated colonic Crohn's disease showed at least one adverse outcome compared with $44 \%$ of patients with ileocolonic Crohn's disease. Furthermore, we were unable to detect an association between any specific, or combination of, major or minor pathological features and outcome in either of the two patient groups, including the presence or absence of perianal disease or granulomas.

The outcome of patients with Crohn's colitis who have had an ileal pouch-anal anastomosis ('pouch') procedure is also controversial. ${ }^{22-24,43,44}$ Some studies show an acceptable low rate of complications, morbidity, and pouch failure, ${ }^{22-24}$ whereas others have shown poor results. ${ }^{43,44}$ In general, pouch complications and failure rates range from 35 to $90 \% .^{22-24,43,44}$ Although some studies have shown better outcome for patients with Crohn's colitis without perianal disease or small bowel involvement, ${ }^{23}$ other studies have not found any clinical or pathological features predictive of outcome after an ileal pouch-anal anastomosis procedure. ${ }^{43}$ In our current study, 20 patients with isolated colonic Crohn's disease and 10 patients with ileocolonic Crohn's disease at initial presentation underwent an ileal pouch-anal anastomosis procedure, because of an incorrect preoperative diagnosis of ulcerative colitis or indeterminate colitis. None of these patients developed anastomotic breakdown or fistulas, both complications that may require pouch diversion or resection. Furthermore, chronic (antibiotic resistant) pouchitis developed in only 15 and $20 \%$ of patients in each of the two groups, respectively. Unfortunately, none of the pathological features, such as the presence or absence of granulomas, perianal disease, fissuring ulcers, transmural inflammation, or granulomas were predictive of pouch complications, including the presence or absence of small intestinal involvement at disease onset. Patients with isolated colonic Crohn's disease showed a similar outcome to those with ileocolonic Crohn's disease. Regardless, these data suggest that many patients with colonic Crohn's disease have good success after the ileal pouch-anal anastomosis procedure, and this may be considered a viable treatment option for patients who are resistant to permanent ileostomy.

Another interesting result of our study is that we identified 16 Crohn's disease patients (14\% overall) in which the colon showed only mucosal 
involvement, without traditional mural features of Crohn's disease, such as transmural lymphoid aggregates, deep or fissuring ulcers, sinus tracts, or fistulas. These cases represented 14 and $13 \%$ of isolated colonic Crohn's disease and ileocolonic Crohn's disease patients, respectively. This subgroup of Crohn's disease patients has been referred to as 'superficial Crohn's colitis' or 'ulcerative colitis-like Crohn's colitis' previously. ${ }^{1,4,19-21,27} \mathrm{We}$ did not identify any clinical, pathological, or outcome features that differed significantly between patients with ulcerative colitis-like Crohn's disease vs those with mural involvement, except for patient age, in which the former group was significantly younger at the time of diagnosis (23 vs 35 years, $P=0.02$ ). In our study, most of the ulcerative colitislike Crohn's disease patients had left-sided colonic involvement $(50 \%)$ and only two $(13 \%)$ had pancolitis. Interestingly, 7 of the $16(44 \%)$ patients had granulomas, and in 1 of these patients, this was the only Crohn's disease-like gross/microscopic feature identified in the colon. However, the outcome of these patients did not differ significantly from those without granulomas, as reported previously by Morpurgo et al. ${ }^{25}$ With regard to outcome, the fact that four of the ulcerative colitis-like Crohn's disease patients had a ileal pouch-anal anastomosis procedure, and none developed complications that required resection of the pouch must be noted. Although four patients developed fistulas, three of those fistulas arose in patients with ileal involvement at initial presentation.

Only two previous studies have evaluated patients with 'superficial' or ulcerative colitis-like Crohn's disease, and, unfortunately, one of these were published only in abstract form. ${ }^{19,20}$ In 1989 , McQuillan and Appelman ${ }^{20}$ described 10 patients with 'superficial Crohn's disease,' characterized by inflammatory changes limited to the mucosa and submucosa. These patients had no, or only minimal, transmural inflammation, and an absence of fissures extending beyond the submucosa. However, unlike our ulcerative colitis-like Crohn's disease patient group, only two of the patients in that study had disease limited 'almost entirely to the colon', whereas the other eight were mostly confined to the small intestine. Furthermore, five of the patients in the study by McQuillan and Appelman had typical transmural Crohn's disease elsewhere in previous resection specimens, and four had typical transmural Crohn's disease elsewhere in the patient's current resection specimen. This is unlike the ulcerative colitis-like Crohn's disease patients in our study in which none had mural involvement (by definition). Harpaz et $a 1^{19}$ also reported 10 cases with superficial Crohn's colitis in abstract form. Those patients were described as being 'grossly similar to ulcerative colitis,' showed involvement of the colon in a continuous manner, and an absence (or near absence) of microscopic inflammation in the superficial submucosa, but unlike our study, those patients were defined by the presence of diagnostic sarcoid-like granulomas. Nevertheless, the patients had a similar mean age at diagnosis as those in our study (26 years), and a high proportion with pancolitis. Two of their cases also had gross and histological involvement of the ileum. Thus, both the results of our study and those of previous small series, suggest that some subtypes of Crohn's disease may affect only the mucosa and superficial submucosa.

In summary, the results of our study demonstrate that patients with isolated colonic Crohn's disease at initial presentation show distinct clinical and pathological features compared with the colon of patients who present with both ileal and colonic involvement. A subgroup of Crohn's disease patients shows involvement of the colon that resembles ulcerative colitis histologically. Further studies should be conducted to determine whether the histology of the colon in patients with colonic Crohn's disease is related to the development of malignancy, molecular phenotype, and specific outcome, on a prospective basis.

\section{Disclosure/conflict of interest}

The authors declare no conflict of interest.

\section{References}

1 Kleer CG, Appelman HD. Surgical pathology of Crohn's disease. Surg Clin North Am 2001;81:159-168.

2 Tanaka M, Riddell RH. The pathologic diagnosis and differential diagnosis of Crohn's disease. Hepatogastroenterology 1990;37:18-31.

3 Peppercorn MA, Odze RD. Colorectal cancer surveillance in inflammatory bowel disease In: Up to Date 2011;19:1.

4 Odze RD. Diagnostic problems and advances in inflammatory bowel disease. Mod Pathol 2003;16: 347-358.

5 Kleer CG, Appelman HD. Ulcerative colitis: patterns of involvement in colorectal biopsies and changes with time. Am J Surg Pathol 1998;22:983-989.

6 Yantiss RK, Farraye FA, O’Brien MJ, et al. Prognostic significance of superficial fissuring ulceration in patients with severe 'Indeterminate' colitis. Am J Surg Pathol 2006;30:165-170.

7 D’Haens G, Geboes K, Peeters M, et al. Patchy cecal inflammation associated with distal ulcerative colitis: a prospective endoscopic study. Am J Gastroenterol 1997;92:1275-1279.

8 Mutinga $\mathrm{M}$, Odze $\mathrm{RD}$, Wang $\mathrm{HH}$, et al. The clinical significance of right-sided colonic inflammation in patients with left-sided chronic ulcerative colitis. Inflamm Bowel Dis 2004;10:215-219.

9 Valdez R, Appelman HD, Bronner MP, et al. Diffuse duodenitis associated with ulcerative colitis. Am J Surg Pathol 2000;24:1407-1413.

10 Haskell H, Andrews CW, Reddy S, et al. Pathologic features and clinical significance of 'Backwash' ileitis 
in ulcerative colitis. Am J Surg Pathol 2005;29: 1472-1481.

11 Lapidus A, Bernell O, Hellers G, et al. Clinical course of colorectal Crohn's disease: a 35 year followup study of 507 patients. Gastroenterology 1998;114: 1151-1160.

12 Lapidus A, Bernell O, Hellers G, et al. Incidence of Crohn's disease in Stockholm county 1955-1989. Gut 1997;41:480-486.

13 Silverberg MS, Satsangi J, Ahmad T, et al. Toward an integrated clinical, molecular and serological classification of inflammatory bowel disease: Report of a Working Party of the 2005 Montreal World Congress of Gastroenterology. Can J Gastroenterol 2005;19: $5 \mathrm{~A}-36 \mathrm{~A}$.

14 Satsangi J, Silverberg MS, Vermeire S, et al. The Montreal classification of inflammatory bowel disease: controversies, consensus, and implications. Gut 2006;55:749-753.

15 Allan R, Steinberg DM, Williams JA, et al. Crohn's disease involving the colon: an audit of clinical management. Gastroenterology 1977;73:723-732.

16 Andrews HA, Lewis P, Allan RN. Prognosis after surgery for colonic Crohn's disease. Br J Surg 1989;76: 1184-1190.

17 Makowiec F, Schmidtke C, Paczulla D, et al. Progression and prognosis of Crohn's colitis. Gastroenterology 1997;35:7-14.

18 Lockhart-Mummery HE, Morson BC. Crohn's disease (regional enteritis) of the large intestine and its distinction from ulcerative colitis. Gut 1960;1:87-105.

19 Harpaz N, Friedman S, George J. Superficial Crohn's colitis: pathological and clinical features including long-term follow up. Mod Pathol 2001;14:86A.

20 McQuillan AC, Appelman HD. Superficial Crohn's disease: a study of 10 patients. Surg Pathol 1989;2:231-239.

21 Soucy G, Schmidt J, Greenberg J, et al. Ulcerative colitis-like Crohn's disease: a clinical/pathologic and outcome study. Gastroenterology 2009;136(s1):S1225.

22 Hartley JE, Fazio VW, Remzi FH, et al. Analysis of the outcome of ileal pouch-anal anastomosis in patients with Crohn's disease. Dis Colon Rectum 2004;47: 1808-1815.

23 Regimbeau JM, Panis Y, Pocard M, et al. Long-term results of ileal pouch-anal anastomosis for colorectal Crohn's disease. Dis Colon Rectum 2001;44:769-778.

24 Panis Y, Poupard B, Nemeth J, et al. Ileal pouch/anal anastomosis for Crohn's disease. Lancet 1996;347: 854-857.

25 Morpurgo E, Petras R, Kimberling J, et al. Characterization and clinical behavior of Crohn's disease initially presenting predominantly as colitis. Dis Colon Rectum 2003;46:918-924.

26 Hancock L, Beckly J, Geremia A, et al. Clinical and molecular characteristics of isolated colonic Crohn's disease. Inflamm Bowel Dis 2008;14:1667-1677.

27 Goldstein N, Sanford W, Bodzin J. Crohn's-like complications in patients with ulcerative colitis after total proctocolectomy and ileal pouch-anal anastomosis. Am J Surg Pathol 1997;21:1343-1353.
28 Singh B, Mortensen NJ, Jewell DP, et al. Perianal Crohn's disease. Br J Surg 2004;91:801-814.

29 Aldhous MC, Drummond HE, Anderson N, et al. Does cigarette smoking influence the phenotype of Crohn's disease? Analysis using the Montreal classification. Am J Gastroenterol 2007;102:577-588.

30 Cosnes J, Cattan S, Blain A, et al. Long-term evolution of disease behavior of Crohn's disease. Inflamm Bowel Dis 2002;8:244-250.

31 Louis E, Michel V, Hugot JP, et al. Early development of stricturing or penetrating pattern in Crohn's disease is influenced by disease location, number of flares, and smoking but not by NOD2/CARD15 genotype. Gut 2003;52:552-557.

32 Smith BR, Arnott ID, Drummond HE, et al. Disease location, anti-Saccharomyces cerevisiae antibody, and NOD2/CARD15 genotype influence the progression of disease behavior in Crohn's disease. Inflamm Bowel Dis 2004;10:521-528.

33 McGovern DP, Hysi P, Ahmad T, et al. Association between a complex insertion/deletion polymorphism in NOD1 (CARD4) and susceptibility to inflammatory bowel disease. Hum Mol Genet 2005;14:1245-1250.

34 McGovern DP, Butler $\mathrm{H}$, Ahmad $\mathrm{T}$, et al. TUCAN (CARD8) genetic variants and inflammatory bowel disease. Gastroenterology 2006;131:1190-1196.

35 Ahmad T, Armuzzi A, Bunce M, et al. The molecular classification of the clinical manifestations of Crohn's disease. Gastroenterology 2002;122:854-866.

36 Fellermann K, Strange DE, Schaffeler E, et al. A chromosome 8 gene-cluster polymorphism with low human beta-defensin 2 gene copy number predisposes to Crohn's disease of the colon. Am J Hum Genet 2006;79:439-448.

37 Wehkamp J, Harder J, Weichenthal M, et al. NOD2 (CARD15) mutations in Crohn's disease are associated with diminished mucosal alpha-defensin expression. Gut 2004;53:1658-1664.

38 Bernell O, Lapidus A, Hellers G. Recurrence after colectomy in Crohn's colitis. Dis Colon Rectum 2001; 44:647-654.

39 Borley NR, Mortensen NJ, Chaudry M, et al. Recurrence after abdominal surgery for Crohn's disease. Dis Colon Rectum 2002;45:377-383.

40 Anseline PF, Wlodarczyk J, Murugasu R. Presence of granulomas is associated with recurrence after surgery for Crohn's disease: experience of a surgical unit. Br J Surg 1997;84:78-82.

41 Wolfson DM, Sachar DB, Cohen A, et al. Granulomas do not affect postoperative recurrence rates in Crohn's disease. Gastroenterology 1982;83:405-409.

42 Chardavoyne R, Flint GW, Pollack S, et al. Factors affecting recurrence following resection for Crohn's disease. Dis Colon Rectum 1986;29:495-502.

43 Braveman JM, Schoetz DJ, Marcello PW, et al. The fate of ileal pouch in patients developing Crohn's disease. Dis Colon Rectum 2004;47:1613-1619.

44 Brown CJ, MacLean AR, Cohen Z, et al. Crohn's disease and indeterminate colitis and the ileal pouch-anal anastomosis: outcomes and patterns of failure. Dis Colon Rectum 2005;48:1542-1549. 